that, even could the mechanical difficulties of construction be overcome, refractors of greater dimensions than the 40-inch cannot be constructed with the hope of uniform performance at all altitudes.

The same journal contains a discussion by Prof. Hartmann of an improvement of the Foucault knife-edge test in the investigation of telescope objectives.

The Temperature of the Sun.-In a preliminary note, now published as an abstract from the Annalen der Physik (vol. xxv., pp. 905-20, 1908), Dr. Goldhammer discusses anew several sets of results obtained in the determination of the temperature of the sun. From the discussion of Langley's various observational data he arrives at the conclusion that the probable actual temperature of the sun is not less than $10,000^{\circ}$ absolute.

The Variation of the Pole.-Prof. Albrecht's annual summary, for 1907 , of the provisional results derived from the observations made at the various international latitude stations appears in No. 4253 of the Astronomische Nachrichten (p. 73, June 5). The extrapolated values for the variation, in the several coordinates, for 1908.o are:$x=-0^{\prime \prime} \cdot 097, y=+0^{\prime \prime} \cdot 185$, and $z=+0^{\prime \prime} \cdot 012$. The curve showing the departure of the pole from the mean position between 1899.9 and 1908.0 shows that the value of the $y$ variation at the commencement of the current year was approaching the maximum observed during that period.

Observations of the Perseid Shower in 1907.-No. 4253 of the Astronomische Nachrichten (p. 83, June 5) contains a note by Prof. J. Sykora in which are given the results of the meteor observations made at Tashkent and Iskander during the nights of August 10-12, 1907; Iskander lies about 44 kilometres to the north-east of Tashkent. From the recorded paths of 178 Perseids the centre of the radiant of the shower, for 1907 , was found to be $\alpha=42^{\circ} \cdot 7, \delta=+53^{\circ} \cdot 8$, and the area covered by the radiants lay between $31^{\circ}$ and $55^{\circ}$ in R.A. and $+49^{\circ}$ and $+59^{\circ}$ in declination. The calculation of the altitudes showed that the mean heights of appearance and disappearance were 167 and 96 kilometres respectively. The trails of nine meteors were photographed, and brief descriptions of trails are given in the paper; several of them indicate marked variations of brightness during the meteor's flight.

\section{RECENT DEVELOPMENTS IN ELECTRIC} $L A M P S$

$\mathrm{I}$ an article which appeared in Nature last June the present writer reviewed briefly some of the improvements which had been made and promised in incandescent electric lamps. At the time that article was written, matters were in a condition of considerable uncertainty on account of the great number of new developments which had been announced, the value of which was, to a very large extent, uncertain. The frequent announcements of these improvements, which were appearing almost weekly, led electrical engineers to feel considerable hesitation in adopting any new lamp for fear that it should be superseded almost immediately after its adoption. Since that time the position has become much quieter, and during the past six months the solid progress which has been made in the introduction of these lamps on the market has been remarkable. Now that considerable experience has been obtained of the practical working value of the different types of lamp, a favourable opportunity is afforded of taking a general survey of the present position and prospects for the future. At the same time, a similar survey may be taken of the conditions existing in the field of arc lighting, in which the developments during the past two or three years have been almost equally noteworthy.

\section{Incandescent Lamps.}

As was pointed out in the article referred to above, the only two lamps which appeared to merit particular attention were the tungsten and the tantalum lamp. The tantalum lamp has now been in practical use for about two years, and has not undergone any appreciable modifi- cation since the time of its first introduction. Difficulties of producing a satisfactory lamp for use with alternating current are still not overcome, and the difficulties of drawing tantalum wire sufficiently fine to enable either low candle-power low-voltage, or medium candle-power highvoltage lamps to be produced still await practical solution. It is true that the range of candle-power with low-voltage lamps has been extended by the introduction of a 16candle-power lamp, and that announcements have been made from time to time that a satisfactory solution has been found for the manufacture of high-voltage lamps. The fact remains, however, that high-voltage lamps are not yet produced commercially. The tantalum lamp has satisfactorily proved its value for electric lighting. Although the general results obtained seem to show a comparatively short average life of about 700 hours, and though the efficiency is approximately only half that of the newer tungsten lamp, it is apparent that this lamp will have to be reckoned with for some time to come as a very important factor in the field of incandescent electric lighting. Though it may not be able to compete on the score of efficiency with the tungsten filament, yet the greater strength of the filament must always operate to counterbalance these disadvantages.

The tungsten lamp, ever since its commercial introduction into this country under the name of the "Osram" lamp by the General Electric Company, has made rapid strides in popularity. Considering that the lamp has only been on the market for a matter of about nine months, its very widespread use at the present time must mark almost a record in the development of electric lighting. In its practical performance, also, this lamp has fulfilled, or more than fulfilled, the hopes which were raised for it before its introduction. Beyond the blackening which occurs with a small percentage of these lamps, the general experience is that a life of 1000 to 1500 hours is obtained almost without any decrease in the initial efficiency of about $\mathrm{x} \frac{1}{4}$ watts per candle. This blackening appears to be a defect in manufacture which will doubtless be soon overcome, since it is not a characteristic of all lamps, but is only observed in a very small percentage, which generally show this defect almost immediately, they are put into use. The tungsten lamp has hitherto possessed the disadvantage, when compared with its tantalum rival, that it could only be used in a vertical position, but a modified type has just been introduced which can be burnt at any angle. On the other hand, the tungsten lamp has the advantage that it is suitable for use on either direct- or alternatingcurrent circuits. Up to the present, the range of voltage for which the lamps can be made is practically the same as that for the tantalum lamps, viz. voltages up to 130 , but the tungsten lamp has not yet been made for voltages of 100 in such low light units as the tantalum lamps, the lowest at present obtainable being approximately thirty candles. Tungsten lamps have also been made, but not yet commercially introduced, for high voltages (200 and above), and the introduction of a 40-candle-power lamp for 200 volts is promised very shortly.

It is yet too early to say precisely what will be the effect of the introduction of these two lamps on electric lighting in general. At present, for the most part probably, the lamps have been used for the lighting of factories, shop windows, and public or semi-public places wherc costs of lighting are very closely considered and artistic effects are of secondary importance. It is perhaps not safe to argue from the success which the lamps have attained in the past year for these purposes that they will meet with corresponding success in private house lighting, especially as in that case the size of the light unit becomes of much greater importance. It is more than probable, however, that the general public will welcome the higher light units if they provide them, as these lamps do, with a means at the same time of actually reducing their lighting bills. The present writer, for example, has substituted two 32-candle-power Osram lamps for a single 16-candle-power carbon filament lamp, and finds that it has resulted in actual saving in money in spite of the fact that four times as much light is obtained. It is noteworthy that the fears which were expressed that the difficulties in running lamps in series would very seriously

No. 2017, vOL. 78] 
affect the introduction of metal filament laups do not appear to have been well founcied.

Beyond these two types. of lamp the development in incandescent lighting has been slight. The exceedingly interesting discovery (from a scientific point of view) by the General Electric Company in America of a method for radically altering the nature of the carbon filament unfortunately came too late to have any practical effect. These so-called "metallised" filament lamps, had they come four or five years ago, would have been welcomed as a great step in advance, but coming as they have at the same time as the metallic filaments, are practically doomed to failure, since they possess the same disadvantages, and, in addition, can only be worked at an efficiency of $2 \frac{1}{2}$ watts per candle. The same may be said of the Nernst lamp, which is almost bound to give way in the sphere of incandescent lighting to metal filament lamps. It is possible that the Nernst lamp will find a sphere of its own for intermediate lighting where light units of roo- to 200-candle-power are required, but it is much more probable that it will be ousted also from this field by the high candle-power metal filament lamps

\section{Arc Lamps.}

The remarkable change in the prospect of incandescent lighting which has been effected by the introduction of the metal filament lamp has been paralleled by a similar change in arc lighting by the introduction of flame lamps. The gain in efficiency in a metal filament lamp over a

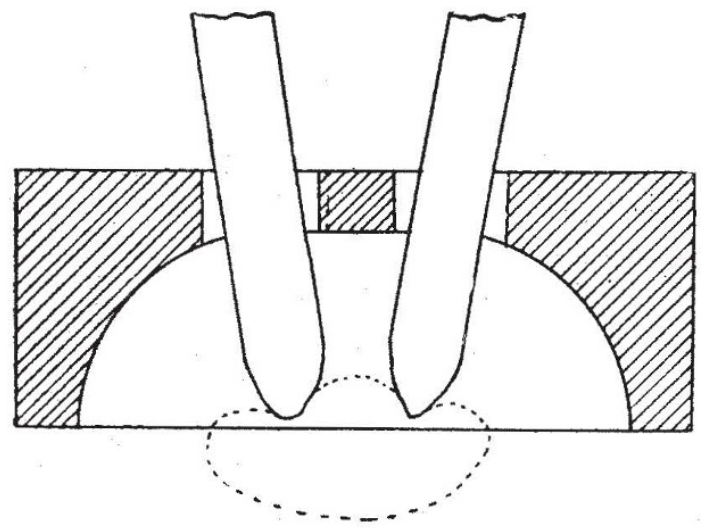

Fig. r.-Arrangement of Carbons in Flame Arc.

carbon filament lamp is approximately three times, and an almost similar gain is obtained in a fiame lamp over an ordinary open type arc lamp. The idea of introducing chemicals into the arc in order to colour the flame is an exceedingly old one, but the practical solution was not obtained until Bremer brought out his mineralised flame carbons. The carbons introduced by Bremer were mineralised carbons in which the flame-producing material was intimately mixed with the material of the carbon rod itself. Alterations were later effected by the various carbon manufacturers by 'which' the flame-producing material, instead of being introduced into the main body of the carbon, was only introduced into the core. This latter type of carbon is by far the most common, but carbons of the Bremer type are still in use, and have been considerably improved recently by $M$. Blondel, who claims to have succeeded by their employment in producing a far more efficient lamp than the ordinary flame lamp.

Flame carbons are constructed to burn with the carbons arranged vertically above one another, as in ordinary lamps, but the more general construction is to arrange the carbons side by side inclined to one another at an angle of about $15^{\circ}$, as shown in Fig. ' $x$. The arc, which balances between the tips of the carbons, as shown in the figure, is spread out into a fan shape, and kept down at the tips by the use of a magnetic controlling field. Immediately above the arc, both in the lamps for vertical and inclined carbons, is placed an inverted cup, the object of which is to prevent the free upward currents of air and maintain the carbons always in an atmosphere of inert gases, thus considerably lengthening their burning hours. This cup is consequently given the name of an economiser.

The arrangement of the carbons in the inclined lamps causes a very large proportion of the light to be thrown vertically downwards, which is certainly not a desirable condition for the lighting of open spaces, involving as it does hanging the lamps very high if even illumination is to be obtained. The rich golden-yellow colour of the light though useful for the purpose of display, is also to be reckoned as a disadvantage of these lamps. The colour is, however, not so very far different from that given by the incandescent lamp, and has not been found a very serious drawback. Flame arcs of other colours, for example, white and pink, can be produced, but the volume of light in these cases is greatly below that of the yellow arc. The flame arc lamp has an efficiency of approximately $0.4-0.5$ watt per mean spherical candle, which is two or three times as good as that of the ordinary opentype arc. The high cost of maintenance in carbons, which are expensive to make and burn rapidly, is very much more than compensated by the low cost of power for a given amount of light. In the Blondel lamp the carbons which are used are of larger diameters, and are arranged vertically one above the other. This has the advantage of giving a better light distribution, and it is claimed that the mean spherical candle-power is nearly double that of the ordinary flame arc.

Whilst the flame arc has been rapidly developed in Europe, it has met with but little success in America on account of the fact that in America the cost of labour for frequent trimming counts much more than it does in this country. For this reason the use of enclosed ares in America has become almost universal in spite of the fact that they only have about. half the efficiency of the ordinary open arc. To meet these somewhat special conditions, experimental work has been carried on during the past two or three years by Messrs. Steinmetz and the General Electric Company of America on the production of a long burning. flame lamp. "The "magnetite" arc, as. it is called, which is the result of this work, utilises electrodes composed chiefly of magnetic oxide of iron. In the latest electrodes titanium oxide, which produces a more intense light in the arc than iron oxide, is used, but iron oxide is still employed to give the electrode conductivity when cold. The actual mixture contains also oxide of chromium, but this plays a purely secondary part in steadying the rate at, which, the other oxides are evaporated.

The lamp is constructed with the magnetite electrode as the negative, and a solid copper electrode as the positive. In place of copper a special alloy is being introduced. The arc has all the characteristics of a flame arc, but possesses some peculiarities due to the fact that the flameproducing material is contained only in the negative electrode. Its efficiency, from figures which have been published, appears to be in the neighbourhood of 0.8 to $\mathrm{I} \cdot 0$ watt per candle, from which it will be seen that it is a little more efficient than the ordinary open-type arc and twice as efficient as the ordinary enclosed arc, which will be its greatest competitor in America. The electrodes, when 12 inches long, are stated to have a life of 150 hours, which is as much or more than similar sized electrodes in enclosed lamps. It will therefore be seen that the magnetite arc is likely to prove a valuable advance on the enclosed arc, and where economical conditions have determined the use of this lamp it is likely to be superseded by the magnetite arc. It cannot, however, at present compete against the ordinary flame lamp, where the cost of trimming is not so important a factor.

\section{Vapour Lamps.}

A word may be said in relation to the development of the mercury vapour lamp. This type of lamp, which has been developed by $\mathrm{Mr}$. Cooper-Hewitt in America, has not met with any extended use on account of the exceedingly unpleasant colour of the light which it gives. Though in some circumstances this may not prevent the adoption of this lamp, there is no doubt that it will always seriously. hamper its use in competition with pleasanter coloured 
illuminants. Attempts have been made to improve the colour by introducing other metals, such as metals of the alkali group, into the tube, but these have not hitherto proved successful... It is stated, however, that a very marked improvement has been effected by the firm of Heraeus, in Germany, by adopting quartz tubes instead of glass tubes, and pushing the temperature at which the arc is run up to very much higher limits. By working the lamp at such a current density that the internal pressure in the tube is approximately one atmosphere, it is stated that the efficiency of the lamp is more than doubled, and that a continuous spectrum is added to the line spectrum of the mercury, thus giving the light a quite pleasant and almost normal colour. These quartz-tube lamps have the additional advantage that under: the conditions of working the length of tube for a given voltage is very much less than when glass tubes are used. The commercial introduction of these lamps is promised for this year.

Reference might also be made to one other type of electric lamp which has during the past year come into commercial use, namely, the Moore tube lamp. This lamp is simply an ordinary vacuum tube of great length, and operated at a very high voltage. The tubes, which are $1 \frac{3}{4}$ inches diameter, can be made up to 200 feet in length, and are fixed up in position by welding together short lengths of tube, high-voltage supply being obtained by means of a transformer the secondary terminals of which are connected to graphite electrodes in the tube. The essential feature of this lamp is the method which has been adopted to overcome the difficulty that the vacuum decreases as the lamp burns, owing to the absorption of gas by the glass tube. In order to maintain the vacuum constant a most ingenious valve is employed. The main tube communicates by means of a branch with the outer air, this branch tube being sealed by a porous carbon plug covered with mercury. The level of the mercury can be altered by the rising or falling of a float; in one position of the float the carbon plug is completely covered, in the other it is partly uncovered. The movements of the float are controlled by a solenoid connected in series with the primary of the transformer supplying the tube. When the vacuum falls the conductivity of the tube increases and the primary transformer current rises; this lifts the mercury float and causes the mercury level to fall, uncovering the point of the carbon plug and allowing a little air to filter into the tube. The conductivity of the tube is thereby decreased, the primary current falls again, and the porous plug is again completely covered with mercury. It is stated that this valve, which operates normally about once a minute, maintains the vacuum in the tube, which is in the neighbourhood of $\mathrm{O} \cdot \mathrm{I} \mathrm{mm}$. of mercury, constant within ro per cent.

The only installation of this lamp in this country is that in the courtyard of the Savoy Hotel, and those who have seen this will probably agree that the light represents in many respects the ideal form of artificial lighting. The colour of the light given by the tube depends upon the gas which it contains, and is pure white for carbon dioxide, slightly pink for nitrogen or air. Nitrogen is stated to be twice as efficient as carbon dioxide, and slightly more efficient than air. When it is desired to operate the tube with either of these gases the open end of the valve is connected to either a phosphorus tube (to extract the oxygen from the air drawn in) or to a gas apparatus generating carbon dioxide by the action of acid on marble. The efficiency of these lamps is difficult to determine, but appears to be in the neighbourhood of 1.6 to 1.8 watts per candle.

It is interesting to note that the improvements described above in incandescent and arc lamps have once again brought electric lighting on practically the same level for cheapness as gas lighting. The introduction of the gas mantle gave gas lighting so great a superiority on the score of cheapness that for a great many years it has only been possible for electricity to hold its own on account of its many other advantages. Just as the ordinary gas mantle beat the carbon filament lamp, the high-pressure gas-mantle systems competed on an equal, or even on a slightly better, basis with arc lighting. The whole complexion which appears is now changed, since the I to $I .5$ watt metal filament lamps can compete with the ordinary mantle for small lighting, and flame lamps are superior to the high-pressure gas lamps. It must bo remembered that, from a scientific point of view, the efficiency of electric lamps is vastly superior to any type of gas lamp, the main cheapness of gas lighting being entirely due to the difference between the cost of power delivered to the lamp in the form of gas and in the form of electric energy. It is interesting to remark that whereas the improvements in gas lighting have been effected by departing from an incandescent flame to an incandescent solid, the improvements in arc lighting have been obtained by a move in exactly the opposite direction.

In conclusion, attention may be directed to the honourable position occupied by this country in the developments of electric lamps. A little more than a century ago an English philosopher, Sir Humphry Davy, discovered the electric arc. Thirty years ago an English inventor, Sir Joseph Swan, shared with an American, Edison, the distinction of overcoming the difficulties attendant upon the production of an incandescent electric lamp of small candlepower. With these two names England's connection with the development of electric lighting begins and ends. The first satisfactory arc-lamp carbons were made by Carré (France). The invention of the cored carbon is due to Siemens (Germany), the practical realisation of flame carbons to Bremer (Germany), and their further development to the Continental manufacturers and to Blondel (France). The magnetite arc has been developed by Steinmetz and the General Electric Company of America. The mercury arc was shown to be practical by. Arons (Germany), and was perfected by Cooper-Hewitt (America). Its latest development is due to the firm of Heraeus (Germany). The vacuum-tube lamp we owe to McFarlaneMoore (America). In incandescent lighting the only radical improvement which has been effected in the carbon filament is the metallised filament of the General Electric Company of America. The Nernst lamp is due to Prof. Nernst and the A.E.G. of Germany. The first metal filament lamp was the "Osmin" lamp of Welsbach (Germany), which was followed by the tantalum lamps of Siemens (Germany) and the tungsten lamps which were perfected by Welsbach (Germany), Just and Hannaman (Austria), and Kuzel (Austria).

One may well ask what is the reason for this unsatisfactory state of affairs. The actual reason is not far to seek. In the field of scientific discovery England has always been, and still is, in the front rank, but not any of the improvements enumerated above are in the nature of discoveries, but are all inventions of a type which have been, and can only be, developed by years of costly experiments carried out always with a commercial end in view, a form of research which is for the most part carried out in the laboratories which are to be found attached to the more important Continental and American factories. The question therefore reduces to asking why it is that these laboratories are not to be found in connection with English works. If you ask the manufacturer, he will probably answer that with unprotected markets, unrestricted competition, and the uncertainty of being allowed to enjoy the fruits of his labour, he cannot afford to spend a share of his money in the prosecution of costly research which may only after many years, and possibly never, yield results. If you ask the man of science, he will probably tell you that it is due to the complicated set of facts which were summed up by Prof. Perry in the words " England's neglect of science," of which the most striking examples are afforded by the position which science takes in all our systems of education, and by the attitude of indifference, amounting almost to contempt, which is directed toward's it by all our Governments, our men of means, our manufacturers, and almost all classes of the community. Each answer probably comprises part of the truth, and the two together possibly comprise the whole, and whilst everyone is agreed that something ought to be done, we are all too. busy arguing which is the best specific to take any steps towards a remedy. In the meantime, further developments are being worked out abroad, and every year is making it harder for this country to make up the leeway which it has lost and is losing.

MAURICE SOLOMON.

NO. 20I7. VOL. 781 